\title{
Prescription/Non-prescription Medicine Misuse and Regulation - Time for a Modern, Fit for Purpose Approach
}

\author{
Mayyada Wazaify ${ }^{1 *}$, Jenny $\operatorname{Scott}^{2}$ \\ ${ }^{1}$ Professor of Pharmacy, Department of Biopharmaceutics and Clinical Pharmacy, Faculty of Pharmacy, The \\ University of Jordan (UJ), Amman, JORDAN.
}

${ }^{2}$ Senior Lecturer of Pharmacy Practice and Medicines Use. Department of Pharmacy \& Pharmacology, University of Bath, UK.

Considering the breadth of medicines available without a prescription and the spectrum of problems that can arise with medication use, pharmacists, particularly those working in community pharmacies, have the potential to make a huge impact on society. Pharmacy like other health professions, has its own codes of ethics for practice, often focused at individual country level. The ownership of such codes is very often internal (where professional bodies regulate their own ethics)e.g. Jordan, but may also be external (where statutory bodies regulate professions) e.g. UK. Such enforced codes of practice must be followed, not only to safeguard the patient and public but also to preserve the integrity of the profession. Nevertheless, violations in practice and ethical standards do happen for many reasons.

An important problem that can raise many ethical issuesand practice challenges is prescription/nonprescription drug misuse and abuse. ${ }^{[1]}$ This problem is prevailing and escalating. ${ }^{[2]}$ Lately, in some Middle Eastern and North Africa (MENA) region countries, the political situation, security issues and closure of borders had led to shortages of illicit drug supplies. ${ }^{[3]}$ With this has come a shift to seek licit and available alternatives from community pharmacies like ophthalmic drugs, ${ }^{[4]}$ pregabalin ${ }^{[5]}$ or oral hypoglycemic agents. ${ }^{[6]}$ In some countries like Jordan, ${ }^{[7]}$ Egypt, ${ }^{[8]}$ Kuwait, ${ }^{\left[{ }^{[0]}\right.}$ and Palestine, ${ }^{[10]}$ addiction is seen to shift from one form of medicine or drug to another and the potential is high for escalation of prevalence. ${ }^{[1]]}$ The main challenges community pharmacists are facing when dealing with addiction to prescription/non-prescription medicines in MENA region countries are: lack of robust legislation, outdated drug classification, and weaknesses in the pharmacy inspection systems. If these factors are not addressed, we predict a continuing rise in prescription/non-prescription medicine addiction.

Although drug classification and laws pertaining to drug dispensing in many countries of the MENAregion are similar to those of the West, these laws are not strictly enforced in community pharmacies in many countries. This contributes to the problem of prescription/nonprescription drug misuse/abuse. Apatient can buy any medication without a prescription, with the exception of controlled narcotics and major tranquilizers (e.g., benzodiazepines), which can only be dispensed after receipt of a prescription signed by a registered physician. ${ }^{[9,12]}$ Such uncontrolled consumption and monitoring of consumption of is one reason of confusion among medications. It also paves the way for some pharmacists to behave unethically and sell medications-that are liable for abuse- without prescription. For example, according to the Jordanian Drug and Pharmacy Practice Law (2013), inspectors from the Jordan Food and Drug Administration (JFDA) frequently visit community pharmacies to check any violation in general and check special prescriptions for medicines, such as opioids, opioid derivatives, or opioid containing preparations classified as Schedules 1-8 drugs. The pharmacist is required to keep a record of these special prescriptions and supply against them, for JFDA inspection. As such, it is a rare incidence for a community pharmacy to sell schedule 1-8products ${ }^{[13]}$ However, some prescriptiononly-medicines that are liable for abuse are not scheduled yet 
in many countries (e.g. pregabalin, performance enhancing hormones, some anticholinergic drugs).This aborts the chance to trace any violation and allows some pharmacists to sell such preparations without a prescription.

Moreover, in community pharmacy, especially in climates of financial crisis, patient care aspects of practice can appear less relevant and very divorced from business aspects. For example, not all private pharmacies in MENA countries maintain patient medication records for prescription or non-prescription (Over-The-Counter; OTC)medicines. ${ }^{[14]}$ They are not legally or professionally required to do so, which makes tracing sale of certain medications difficult. Against a pressure to generate income, the temptation to exploit those dependent on substances seeking prescription medicines to misuse/abuse by selling products at vastly inflated prices, becomes apparent.

Pharmacists may however, also be contributing to this problem unknowingly, by allowing repeat supply of medicines that were legitimately prescribed in the past. An example may be a short course of benzodiazepines prescribed for anxiety. Repeat supply and prolonged use, facilitated by repeat sales, can turn into dependence and an inability to cease use. More research is needed to understand why pharmacists take it upon themselves to facilitate such repeat supply, as it may be more complex than the single factor of income generation.

An additional contributing factor to the inappropriate supply of prescription/non-prescription medicines is lack of training for pharmacists at undergraduate level. In many schools of pharmacy, the dependence potential of medicines, models of addiction and addiction treatment are barely covered in the curriculum. Pharmacists may consequently lack confidence in responding to patients with an apparent or emerging dependence on prescription medicines. This coupled with a lack of referral pathways into care may mean persistent supply of medicines fueling the development or sustainment of dependence on such medicines. People using medicines in a dependent way are likely to be at high risk of side effects and may experience a lot of harm related to these medicines. Pharmacists are well placed in the community to offer advice to such people but sadly often lack the skills to identify or respond to such concerns.

Another contributing factor that we have identified in our work is the culture and cohesion between people within the MENA region of the world. Pharmacists, like others, may feel the obligation and desire to help an acquaintance or regular patient with their needs (in this case supply medicines liable to abuse) despite concerns around dependence. Factors such as age, gender, family dynamics, job status can all be barriers to refusing to supply a medicine if the requestor is perceived to have higher status. This is likely to be a very challenging aspect to address.

In summary, the general public think highly of the pharmacy profession and seek advice from pharmacists about a range of health concerns on a daily basis. Pharmacists need to ensure the safe and appropriate use of medicines, this is enshrined in codes of ethics across the globe. It is however unsurprising that in time of conflict, financial crisis, displacement of millions of people and strains on natural resources that some members of the public should turn to psychoactive substances to relieve their distress and trauma. A common source of such psychoactive substances includes prescription and non-prescription medicines. This makes pharmacists knowingly or unknowingly, dealers in such medicines. In order to 'fix' this problem, many facets need to be addressed. The education of undergraduate pharmacy students should include awareness of substance dependence and ways in which it can be controlled and patients supported. Pharmacists should be able to give harm reduction advice and signpost suspected addicts to treatment services. The regulation of psychoactive medicines needs to be modernized, fit for purpose and tightened to ensure supply is traceable and pharmacists are accountable for the medicines that they issue. Tighter inspection and law enforcement is needed, for example to prevent the practice of repeat issuing of medicines against a previously spent prescription. In summary, there is a lot to do before the pharmacy profession can distance itself from being the dealer of many medicines liable to abuse.

\section{REFERENCES}

1. Hughes GF, McElnay JC, Hughes CM, McKenna P. Abuse/misuse of nonprescription drugs. Pharm World \& Sci. 1999;21:251-255.

2. Cooper RJ. Over-the-counter medicine abuse - a review of the literature. J Subst Use. 2013;8(2):82-107.

3. Kravitz M, Nichols W. A Bitter Pill to Swallow: Connections Between Captagon, Syria, and The Gulf. J Int Affairs. 2016;69:2.

4. Wazaify M, Alali M, Yousef M, Alqammaz S. Ophthalmic Drops Abuse in Community Pharmacy Setting: A Cross-Sectional study from Jordan. J Substance Use. 2017; 1-6.

5. Al-Husseini A, Wazaify M, Van Hout MC. 2017. Pregabalin Misuse and Abuse in Jordan: a Qualitative Study of User Experiences. Int J Mental Healt Addiction. 2017:1-13.

6. Kalbani RJ, Wazaify M. Prevalence of prescription and non-prescription drugs-related overdoses at emergency departments in Amman-a cross sectional study. J Substance Use. 2017;22(1):77-82

7. Albsoul-Younes A, Wazaify M, Yousef A, Tahaineh L. Abuse and misuse of prescription and nonprescription drugs sold in community pharmacies in Jordan. Substance Use and Misuse. 2010;45(9):1319-29.

8. Jousilahti P, Madkour S, Lambrechts T, Sherwin E. Diarrhoeal disease morbidity and home treatment practices in Egypt. Public Health.1997;111:5-10. 
Wazaify and Scott: Prescription/Non-prescription Medicine Misuse and Regulation -Time for a modern, fit for Purpose Approach

9. Matowe L, Al-Kandery A, Bihzad S. Pharmacy in Kuwait. Am J Health System Pharm. 2003;60:1591-2.

10. Sweileh W, Arafat R, Al-Khyat L, Al-Masri D, Jaradat N. A pilot study to investigate over-the-counter drug abuse and misuse in Palestine. Saudi Med J. 2004;25:2029-32.

11. Wazaify M, Abood EA, Tahaineh L, Albsoul-Younes AM. Jordanian community pharmacists' experience regarding prescription and nonprescription drug abuse and misuse in Jordan- An update. J Substance
Use. 2016;22(5):463-8

12. Al-Wazaify M, Albsoul-Younes A. Pharmacy in Jordan. Am J Healt System Pharm. 2005;62:2548-51.

13. Jordan Food and Drug Administration. Instructions for Prescribing and Medical Records. 2017. Available from: http://www.jfda.jo/EchoBusV3.0/ SystemAssets/PDF/AR/LawsAndRegulation/Drug/RegisterSection/2017.pdf

14. Fathelrahman $\mathrm{Al}$, Ibrahim $\mathrm{MI}$ and Wertheimer Al. 'Pharmacy practice in developing countries', London: Elsevier; 2016. ISBN 9780128017142

Received: 1 October 2017; Accepted: 25 October 2017

*Correspondence to:

Prof. Mayyada Wazaify, PhD, Department of Biopharmaceutics and Clinical Pharmacy, Faculty of Pharmacy, The University of Jordan, Amman-11942, JORDAN

Email:m.wazaify@ju.edu.jo

Copyright: (C) the author(s),publisher and licensee Indian Academy of Pharmacists. This is an open-access article distributed under the terms of the Creative Commons Attribution Non-Commercial License, which permits unrestricted noncommercial use, distribution, and reproduction in any medium, provided the original work is properly cited.

Cite this article as: Wazaify M, Scott J. Prescription/Non-prescription Medicine Misuse and Regulation - Time for a modern, fit for Purpose Approach. J Pharm Pract Community Med. 2017;3(4):197-9. 